\title{
Model Predictive Control Strategies for Constrained Soft Landing on an Asteroid
}

\author{
Dominic Liao-McPherson, William Dunham, and Ilya Kolmanovsky*
}

\begin{abstract}
In this paper, several autonomous control strategies for soft landing on an asteroid are developed and compared. These strategies exploit prediction and onboard optimization. They include a non-linear model predictive controller which directly handles the nonlinear model of the spacecraft dynamics and constraints. A convex model predictive controller which is based on the linearized model and an extended command governor in combination with an inner loop feedback controller. An input observer is used in all cases to compensate for errors between the estimated and actual gravity models. A two phase approach is used which divides the maneuver into a circumnavigation and a landing phase. Simulations performed on a full nonlinear model of the spacecraft near the asteroid Eros with measured gravity parameters demonstrate successful landings for all three control schemes.
\end{abstract}

\section{Nomenclature}

$\Delta T \quad$ Sampling period

$\dot{x}, \dot{y}, \dot{z}$ Spacecraft position and velocity expressed in the asteroid frame

$\eta \quad$ Normal vector to a hyperplane

$\mathcal{N}\left(m, \theta^{2}\right)$ Normal distribution with mean $m$ and variance $\theta^{2}$

$\mathbf{O}_{n a} \quad$ Direction cosine matrix relating frames A and $\mathrm{N}$

$\overrightarrow{\mathcal{F}_{A}} \quad$ Asteroid fixed reference frame

$\overrightarrow{\mathcal{F}_{N}} \quad$ Asteroid surface normal fixed reference frame

$\xi \quad$ Spacecraft state expressed in asteroid fixed frame

$A, B \quad$ Discrete linear dynamics

$a, b, c$ Constant density ellipsoid model semi-major axes

$A_{c}, B_{c}$ Continuous linear dynamics

$a_{s}, b_{s}, c_{s}$ Safety ellipsoid semi-major axes

$C_{l, m}, S_{l, m}$ Stokes Coefficients

$F \quad$ Gravitational force exerted by the asteroid

$G \quad$ Gravitational potential of the asteroid

$R_{0} \quad$ Reference radius for stokes coefficients

$u_{x}, u_{y}, u_{z}$ Spacecraft thrust expressed in asteroid frame

$x, y, z$ Spacecraft position and velocity expressed in the asteroid frame

$S_{++} \quad$ Cone of positive definite symmetric matrices

$S_{+} \quad$ Cone of positive semidefinite symmetric matrices

\section{Introduction}

There is a growing interest in sending spacecraft to asteroids and other small stellar bodies. The Rosetta mission has been studying the comet 67P/Churyumov-Gerasimenko since 2014 where the Philae lander successfully reached the surface [1]. NASA's OSIRIS-REx mission is scheduled to launch near the time of this publication with the goal of collecting samples off the surface of the asteroid 101955 Bennu and returning to Earth [2].

\footnotetext{
*Department of Aerospace Engineering, University of Michigan, Ann Arbor, MI
} 
The asteroid environment posses many challenges for spacecraft missions in close proximity [3]. The shape and gravity of such bodies can be complex [4] and difficult to determine without long-term observations. The large distances between these bodies and the Earth also creates a time-delay for ground based GNC. Consequently, a spacecraft must rely on onboard autonomous control systems to accomplish some of its objectives. Furthermore, the relatively large thrust authority possessed by a spacecraft relative to the gravity of such bodies allows for the applications of feedback control, provided adequate measurements can be made.

Traditionally, missions seeking to land on the surface of such bodies have done so by tracking a precomputed trajectory. This method can guarantee mission safety by having the spacecraft enter a safe mode if constraints are in danger of being violated [5]. Ruoyan et al.[6] examined the feasibility for using a Model Predictive Controller (MPC) for tracking such reference trajectories. Lee et al. [7] presented a control scheme for reaching a desired position and orientation for hovering near an asteroid in finite-time. Yang and Baoyin [8] developed a method to determine a fuel optimal trajectory for a controlled soft landing using a homotopy approach. These methods are effective if the mission has sufficient time to characterize the asteroid's environment. If the gravity field and the shape of the asteroid are not well known, open-loop trajectories may not be able to guarantee a safe and efficient trajectory for the spacecraft.

In a recent paper [9], the authors considered a spacecraft landing on an asteroid using a constant density ellipsoid approximation for the gravity model. A controller was devised that uses a Linear Quadratic Regulator (LQR) for stabilization to a target point, an Extended Command Governor (ECG) for constraint enforcement by varying the reference to the LQR, and an input observer for model error disturbance rejection. A method of rotating hyperplanes was used to convexify the collision avoidance constraints as in Petersen et al.c[10] and Weiss [11]. Note however, that Jewison et al. [12] have shown that this method can lead to sub-optimal trajectories due to the hyperplanes blocking out potential paths from the spacecraft.

This paper compares the performance of three different predictive control strategies applied to the problem of autonomously landing a spacecraft on Eros. The controllers are an extended command governor supervising an inner loop LQR controller, a convex model predictive control (CMPC) method, which uses a linear model, and a nonlinear model predictive controller (NMPC). All three designs use variations of the two phase landing approach and an input observer from [9] but the constraints for the CMPC and NMPC controllers are reformulated to take advantage of the increased capabilities of the convex and nonlinear programming solvers, respectively. In addition, all three techniques make use of input observations to compensate for the gravity model errors. This contrasts with other work on constrained proximity operations which do not explicitly consider uncertain disturbance forces. Nonlinear simulation results are specific to the asteroid 433 Eros and take advantage of the well known gravity [13] and shape [14] characterizations of the body.

The paper is organized as follows. Section II introduces the equations of motion with emphasis on the gravity model of the asteroid 433 Eros and the models used by the controllers. Section III provides an overview of the landing strategy which is common to all three controllers. Section IV describes the controllers in detail including the input observer used to help reject disturbances; detailed mathematical expressions for the constraints; and the design of the ECG, CMPC, and NMPC controllers. Simulation results are presented in section $\mathrm{V}$ and concluding remarks are made in section VI.

\section{Model}

In this section, a model of the dynamics of a spacecraft in the sphere of influence of a small body are discussed. The spacecraft is assumed to be equipped with six ideal thrusters, to be able to generate propulsive forces in all directions. The asteroid itself is rotating at a constant angular rate about the principal axis

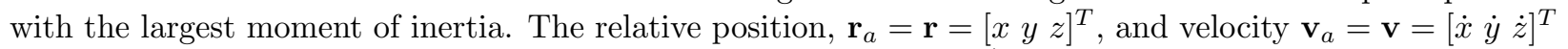
of the spacecraft expressed in an asteroid fixed frame (denoted by $\overrightarrow{\mathcal{F}}_{A}$ using vectrix notation [15]) the origin of which is at the center of mass of the asteroid, are governed by the following differential equations,

$$
\begin{gathered}
\ddot{x}=F_{g, x}+2 n \dot{y}+n^{2} x+u_{x}, \\
\ddot{y}=F_{g, y}-2 n \dot{x}+n^{2} y+u_{y}, \\
\ddot{z}=F_{g, z}+u_{z},
\end{gathered}
$$

where $n$ is the rotation rate of the asteroid, $F_{g, x}, F_{g, y}$ and $F_{g, z}$ are the components of the gravitational force per unit mass exerted on the center-of-mass of the spacecraft by the asteroid, and $u_{x}, u_{y}$, and $u_{z}$ are the 
control forces per unit mass. The rotation rate of the asteroid is assumed to be known as, under certain conditions, it can be determined from the Earth through the use of light curve analysis [16]. The gravity force can be calculated from the potential, given as a summation of spherical harmonics

$$
G=-\frac{\mu}{R_{0}} \sum_{l=0}^{\infty} \sum_{m=0}^{n}\left(C_{l, m} V_{l, m}+S_{l, m} W_{l, m}\right),
$$

where $\mu$ is the asteroids gravitational constraint; $R_{0}$ is the radius of a reference sphere; $C_{l, m}$ and $S_{l, m}$ are the Stokes coefficients; and $V_{l, m}$ and $W_{l, m}$ are functions of position calculated using the Montenbruck recursion scheme [17]. The gravity force is then calculated as the gradient of the potential

$$
\mathbf{F}=\left[\begin{array}{llll}
F_{g, x} & F_{g, y} & F_{g, z}
\end{array}\right]^{\mathrm{T}}=-\nabla G .
$$

Note that direct measurements over a long period of time are required to determine the Stokes coefficients in (4) for a small nonuniform body. Several alternative also exist to estimate the coefficients. A constant density polyhedron can be used to directly calculate the gravity potential, but requires a sophisticated shape model of the object [18]. Stokes coefficients can also be found by filling an object with point masses to simulate the total gravity field [4]. In this paper, a constant density ellipsoid approach, as described in Reference [4], is used in the controller design (see Section II.A) while a higher fidelity model is used for simulations. The Stokes coefficients for the gravity model used in simulations are obtained from measurements reported by the NEAR-Shoemaker mission [19].

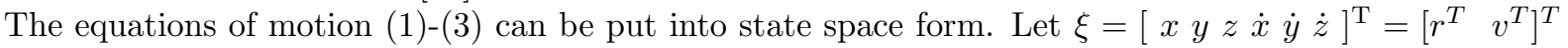
be the relative state vector, $u=\left[\begin{array}{lll}u_{x} & u_{y} & u_{z}\end{array}\right]^{\mathrm{T}}$ be the control vector, and $t$ be the current time. Then the equations can be written in the following affine in controls form,

$$
\dot{\xi}(t)=A_{c} \xi(t)+F_{g}(\xi(t))+B_{c} u(t) .
$$

\section{A. Prediction Models}

This section describes the prediction models used by all three controllers.

\section{Nonlinear Prediction Model}

The nonlinear prediction model, based on the plant (6), is used to compute an optimal control in the NMPC approach. The controller replaces the high-fidelity gravity model $F_{g}(\xi)$, which may not be available without extensive observations of the asteroid, with a low fidelity approximate model $\hat{F}_{g}(\xi)$. The approximate model is based on a 4th order truncation of (4), the coefficients of which are computed using a constant density ellipsoid approach. Specifically, the asteroid is approximated using an ellipsoid with the form

$$
\left(\frac{x}{a}\right)^{2}+\left(\frac{y}{b}\right)^{2}+\left(\frac{z}{c}\right)^{2}=1
$$

The symmetry of the body allows the Stokes coefficients to be calculated with relative ease. All $S_{l, m}$ coefficients in (4) are zero and the $C_{l, m}$ coefficients can be calculated up to the fourth order as [4],

$$
\begin{gathered}
C_{20}=\frac{1}{5 R_{0}^{2}} \frac{c^{2}-\left(a^{2}+b^{2}\right)}{2}, \\
C_{22}=\frac{a^{2}-b^{2}}{20 R_{0}^{2}}, \\
C_{40}=\frac{15}{7}\left(C_{20}^{2}+2 C_{22}^{2}\right), \\
C_{42}=\frac{5}{7} C_{20} C_{22}, \\
C_{44}=\frac{5}{28} C_{22}^{2} .
\end{gathered}
$$

The resulting continuous time prediction model can be written as

$$
\dot{\xi}(t)=A_{c} \xi(t)+\hat{F}_{g}(\xi(t))+B_{c} u(t)=f_{c}(\xi(t), u(t)) .
$$




\section{Linearized Prediction Model}

A linearized prediction model is used for CMPC and for the ECG designs. This model is obtained by linearizing the nonlinear prediction model (13) about the current state estimate $\xi_{0}=\hat{\xi}(t)$ and $u_{0}=0$ in the case of CMPC or about the target point $\left(\xi_{T}, u_{T}\right)$ for the ECG. The linearized equation in the ECG case may be written as

$$
\delta \dot{\xi}(t)=\left(A_{c}+\left.\frac{\partial \hat{F}_{g}}{\partial \xi}\right|_{\xi_{0}, u_{0}}\right) \delta \xi(t)+B_{c} \delta u(t)=A_{l} \delta \xi(t)+B_{c} \delta u(t)
$$

where $\delta \xi(t)=\xi(t)-\xi_{0}$ and $\delta u(t)=u(t)-u_{0}$.

\section{Measurement Uncertainty}

Measurement uncertainty is simulated by corrupting the state estimates used for computing controls by additive Gaussian noise. The position error $\sigma_{r}(t)$ is drawn from a Gaussian distribution with zero mean and a variance of a third of a meter, this approximately corresponds to a three sigma confidence level that the position error is within 1 meter i.e., $\sigma_{r}(t) \sim \mathcal{N}\left(0,(1 / 3)^{2}\right)$. Similarly, the three sigma confidence level for the velocity error $\sigma_{v}(t)$ is assumed to be half a meter per second; i.e., $\sigma_{v}(t) \sim \mathcal{N}\left(0,(0.5 / 3)^{2}\right)$.

\section{Landing Mission Design}

In order to achieve the goal of landing at a target area on the surface of the asteroid we seek to stabilize the spacecraft to a point, $\mathbf{r}_{L} \in \mathbb{R}^{3}$, five meters above the asteroid surface (see Figures $1 \mathrm{~b}, 6 \mathrm{~b}$, and $6 \mathrm{a}$ ). The mission is divided into two phases: a circumnavigation phase and a landing phase. The phases differ by the set-point target and constraints being enforced. In both of these phases, a control constraint is imposed as

$$
u_{\min } \leq u(t) \leq u_{\max },
$$

to account for thruster saturation limits.

\section{A. Circumnavigation Phase}

When the spacecraft is far from the landing zone, it must circumnavigate the asteroid while avoiding collisions with the surface. To avoid collisions with the surface an exclusion constraint ellipsoid that envelops the asteroid is introduced. Mathematically, the constraint is expressed as

$$
c_{s e}=1-\left(\frac{x(t)}{a_{s}}\right)^{2}+\left(\frac{y(t)}{b_{s}}\right)^{2}+\left(\frac{z(t)}{c_{s}}\right)^{2} \leq 0
$$

This type of ellipsoidal constraint has been successfully used by Jewison, et al. [12]. This nonconvex collision avoidance constraint is handled directly by the nonlinear MPC controller and it is convexified by using a rotating hyperplane method in the ECG and CMPC approaches.

The target point in the circumnavigation phase is $\mathbf{r}_{e}$, which lies one-hundred meters above the safety ellipsoid directly above the target point $\mathbf{r}_{L}$ at the point where the landing point surface normal intersects the safety ellipsoid.

The rotating hyperplane constraint for the ECG and the CMPC is set-up as follows. A hyperplane intended to separate the spacecraft from the ellipsoid is rotated along the surface of the ellipsoid at a constant rate, beginning at the point on the ellipsoid closest the spacecraft at time $k \Delta T$, denoted as $\mathbf{r}_{1}$, and ending when the hyperplane reaches $\mathbf{r}_{e}$ where $\Delta T$ is the sampling period of the controller and $k$ is the discrete time index. The rotating hyperplane constraint is formed at time instant $j \Delta T$ along the prediction horizon as follows

$$
\begin{gathered}
\eta_{j}^{\mathrm{T}}\left(H \xi((k+j) \Delta T)-\mathbf{r}_{j}\right) \geq 0, \quad j=1,2, \ldots N_{\eta}-1 \\
\eta_{j}^{\mathrm{T}}\left(H \xi((k+j) \Delta T)-\mathbf{r}_{n_{\eta}}\right) \geq 0, \quad \forall j \geq N_{\eta}
\end{gathered}
$$

where $\eta_{j} \in \mathbb{R}^{3}$ is the normal to the hyperplane at time $(k+j) \Delta T$ pointing outward from the ellipsoid, $N_{\eta}$ is the constraint horizon, $\mathbf{r}_{j}$ defines the point of intersection between the hyperplane and the safety ellipsoid, and $H=\left[\begin{array}{ll}I_{3} & 0\end{array}\right]$. Note that since the hyperplane finishes its rotation at $\mathbf{r}_{e}, \mathbf{r}_{n_{\eta}}=\mathbf{r}_{e}$ if the horizon 
is sufficiently long. The rotating hyperplane constraint is visualized in Figure 1b, where the cross denotes the position of the spacecraft.

The safety ellipsoid and the rotating hyperplane are shown in Figures 1a and 1b, respectively.

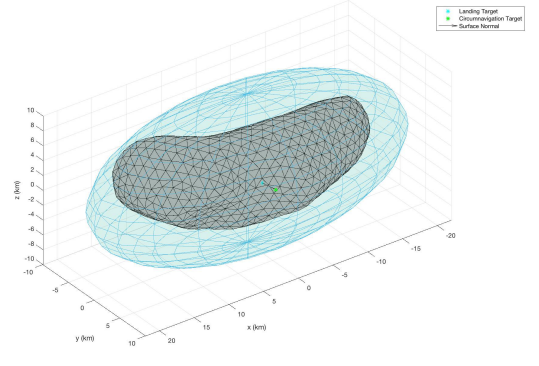

(a) Safety Ellipsoid

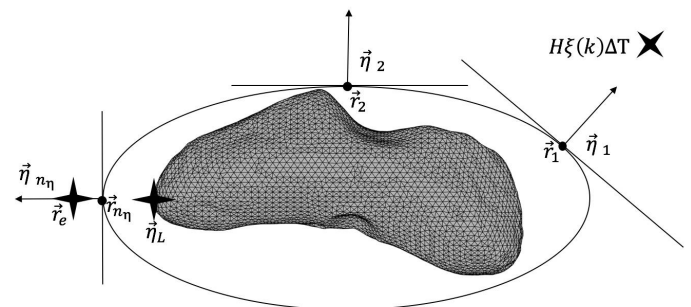

(b) Rotating hyperplane constraint

Figure 1: Circumnavigation Constraints

\section{B. Landing Phase}

Once the spacecraft has reached a pre-determined distance, $d_{\text {switch }}$, from $\mathbf{r}_{e}$ the circumnavigation phase ends. The spacecraft controller then switches to the landing phase. The new target becomes $\mathbf{r}_{L}$. The nonlinear equations of motion are linearized about $\xi_{0}=\left[\begin{array}{llll}\mathbf{r}_{L} & 0 & 0 & 0\end{array}\right]^{\mathrm{T}}$ and used to design the input observer and the inner loop of the ECG controller.

To prevent a collision during landing, the spacecraft is constrained to remain inside a predefined paraboloid, as shown in Figure 2, during descent. For both the CMPC and NMPC approaches the nonlinear but convex parabolic constraint is applied directly. In the ECG case the landing parabola is approximated by a four-sided pyramid in order to replace the nonlinear constraint with linear constraints compatible with a quadratic programming solver.

The landing paraboloid may be specified as follows; let $\mathbf{n}$ be the asteroid surface normal evaluated at the landing site $\mathbf{r}_{L}$ and let $\overrightarrow{\mathcal{F}}_{n}$ be a frame the vertical axis of which is aligned with the surface normal (i.e., $\mathbf{n}_{n}=\left[\begin{array}{lll}0 & 0 & 1\end{array}\right]^{T}$ ). If $\mathbf{O}_{n a}$ is the direction cosine matrix (DCM) relating the two frames (i.e., $\overrightarrow{\mathcal{F}}_{n}=\mathbf{O}_{n a} \overrightarrow{\mathcal{F}}_{a}$ ) the landing parabola constraint can be expressed as

$$
c_{l p}=\left(\mathbf{r}-\mathbf{r}_{p}\right)^{T} \mathbf{O}_{n a}^{\mathrm{T}}\left[\begin{array}{ccc}
\frac{1}{a_{p}^{2}} & 0 & 0 \\
0 & \frac{1}{a_{p}^{2}} & 0 \\
0 & 0 & 0
\end{array}\right] \mathbf{O}_{n a}\left(\mathbf{r}-\mathbf{r}_{p}\right)-\mathbf{n}_{n}^{\mathrm{T}} \mathbf{O}_{n a}\left(\mathbf{r}-\mathbf{r}_{p}\right) \leq 0,
$$

where $a_{p}$ is a parameter which controls the width of the paraboloid and $\mathbf{r}_{p}=\mathbf{r}_{L}-d_{p} \mathbf{n}$; this shifts the paraboloid slightly into the asteroid and relaxes the constraint to prevent the feasible region shrinking to a point at the landing target which can cause numerical issues.

The ECG uses a pyramid defined by four normals $\sigma_{A}, \sigma_{B}, \sigma_{C}, \sigma_{D} \in \mathbb{R}^{3}$. The tip of the pyramid is the point $\mathbf{r}_{p}$. If the spacecraft is within the pyramid at time $k \Delta T$, then the constraints imposed on the spacecraft over the prediction horizon are given by

$$
\begin{gathered}
\sigma_{i}^{\mathrm{T}}\left(H \xi((k+j) \Delta T)-\mathbf{r}_{L}-d\right) \geq 0, \\
i=A, B, C, D, \quad j=0,1, \cdots, j^{*},
\end{gathered}
$$

$j^{*}$ is the horizon length over which the constraint is imposed, and where $H$ is defined as in the circumnavigation phase above. Visually, this is seen in Figure 3b, where the cross is the position of the spacecraft.

To prevent the spacecraft from overshooting the target and making contact with the asteroid at high velocity, a hyperplane constraint is used to prevent overshoot. This constraint is shown in figure 3a and is expressed mathematically as

$$
c_{n o}=-\mathbf{n} \cdot\left(\mathbf{r}-\mathbf{r}_{T}\right) \leq 0,
$$




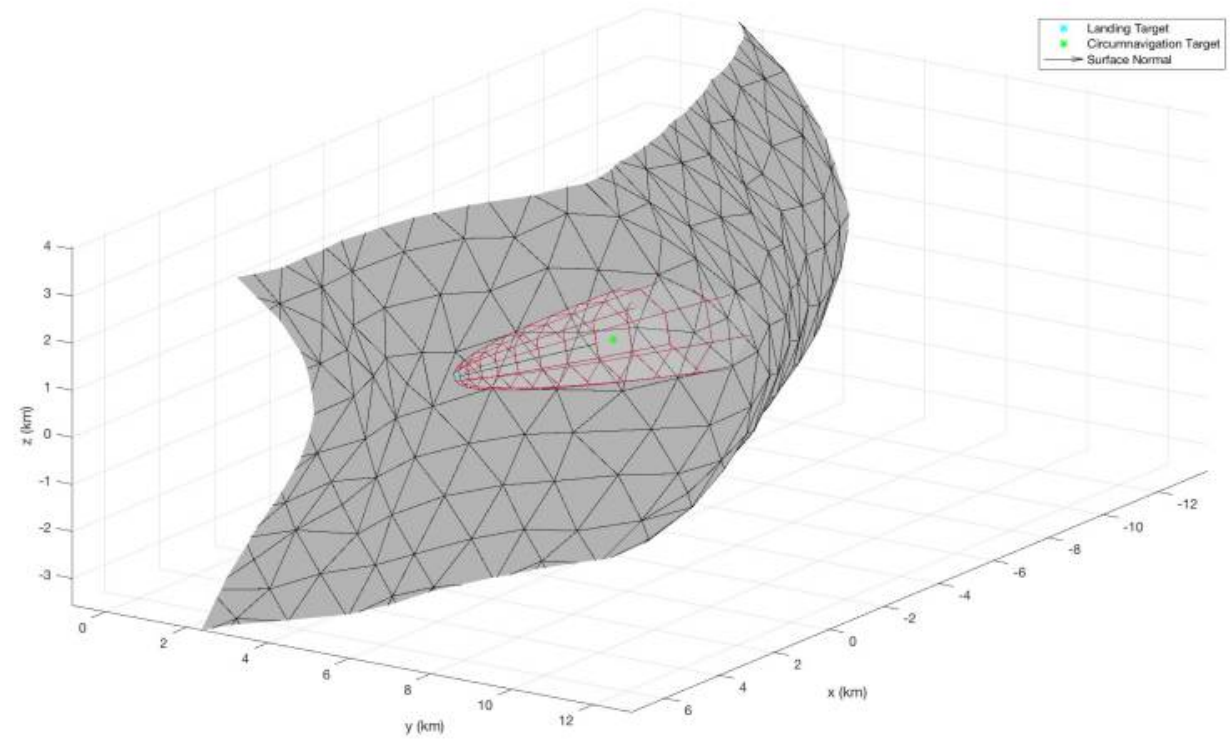

Figure 2: The landing parabolic constraint. Note that the landing target which is positioned $5 \mathrm{~m}$ above the surface.

where we have chosen $\mathbf{r}_{T}$ to be 2 meters above the surface. Note that (20) is a linear constraint which can be applied directly to all three control schemes.

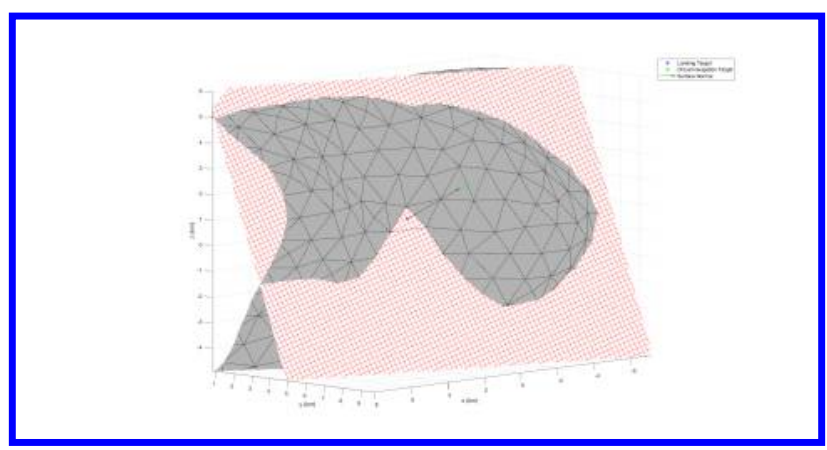

(a) The no overshoot constraint

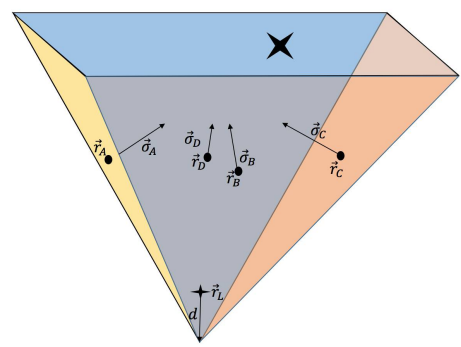

(b) The pyramid approximation of the parabolic landing constraint

Figure 3: No overshoot and landing pyramid constraints

\section{Controller Design}

This section describes the controller architectures used to control the spacecraft. First, the input observer used to counteract the model error disturbances is introduced and then the ECG, NMPC, and CMPC controllers are described.

\section{A. Input Observer}

To account for the mismatch in the gravity model based on (8)-(12) and the actual gravity of the asteroid, an input observer based upon [20] is used. For this, consider (14) modified as

$$
\delta \dot{\xi}(t)=A_{l} \delta \xi(t)+B_{c}(\delta u(t)+w(t)),
$$


where the disturbance $w(t)$ represents the mismatch (at the level of forces) between the assumed linear model of the dynamics and the actual dynamics. Note that the disturbance is matched to the input of the system. To counteract $w(t)$, the control input is augmented with a disturbance estimate,

$$
\delta u(t)=\delta u(t)_{c l}-\hat{w}(t)
$$

where $\delta u(t)_{c l}$ is the difference between the closed-loop control input of a controller and the nominal control at the target point,

$$
\delta u(t)_{c l}=u(t)_{c l}-u_{0}
$$

such that the disturbance $w(t)$ is counteracted by its estimate $\hat{w}(t)$.

The estimate in the input observer is determined as follows. Let $z(t)$ be defined as an output that reflects spacecraft velocities,

$$
z(t)=H_{O} \delta \xi(t)
$$

where $H_{O}=\left[\begin{array}{ll}0_{3} & I_{3}\end{array}\right]$. We assume that this measurement is available to us from either instruments or a separate on-board estimator. Following [20], we define an auxiliary variable $\zeta(t)$ that obeys the following dynamics

$$
\dot{\zeta}(t)=\gamma\left(\hat{g}(t)+H_{O} A_{l} \delta \xi(t)+H_{0} B_{c} \delta u(t)\right),
$$

where $\gamma$ is the gain of the observer. The variable $\hat{g}(t)$ is defined by

$$
\hat{g}(t)=\gamma z(t)-\zeta(t)
$$

and is used to determine the estimate of the disturbance,

$$
\hat{w}(t)=\left(H_{O} B_{c}\right)^{L} \hat{g}(t)
$$

where $\left(H_{O} B_{c}\right)^{L}$ is the left inverse of the matrix $H_{O} B_{c}$, where for a matrix $M, M^{L}=\left(M^{\mathrm{T}} M\right)^{-1} M^{\mathrm{T}}$. It is proven in [20] that if a bound $\bar{b}$ exists on the rate of change of the disturbance such that

$$
\bar{b}^{2}>\sup _{t \geq 0} \dot{w}(t)^{\mathrm{T}} \dot{w}(t)
$$

then over time the estimation error $w(t)-\hat{w}(t)$ will converge to a neighborhood of the origin. The size of this neighborhood is determined by the observer gain $\gamma$, and can be made arbitrary small for sufficiently large values of $\gamma$. The intended effect of the input observer is that the dynamics experienced by the controller are rendered closer to the model for which the controller is derived. Even with some noise in the measurements, the input observer is able to reduce the size of the offset from the target point in steady state.

\section{B. Extended Command Governor}

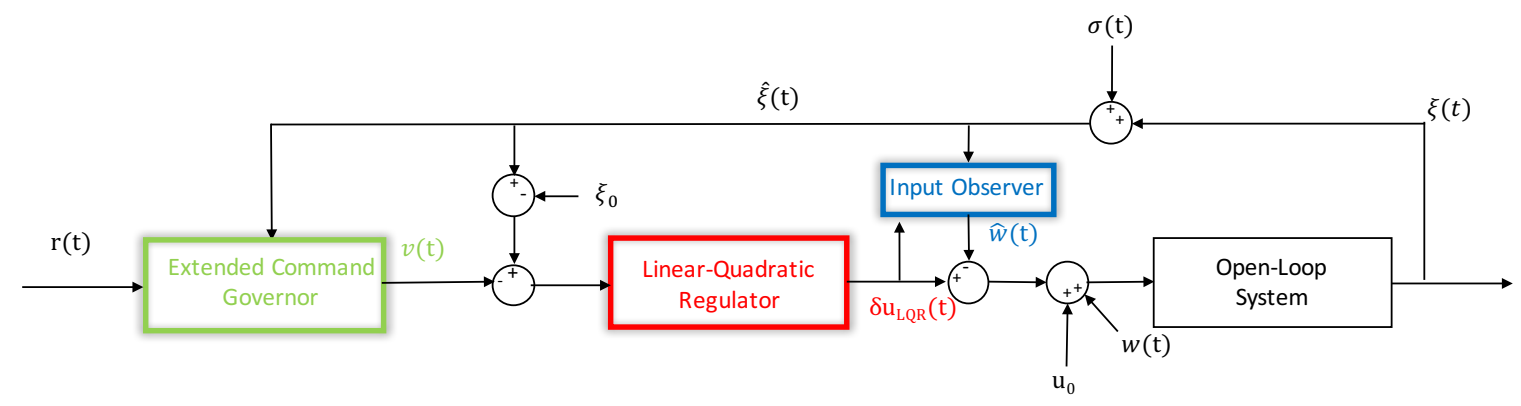

Figure 4: The ECG control strategy

The control scheme from [9] consists of three loops, shown in Figure 4. The inner-most loop contains the input observer as explained above. The middle loop contains a standard linear feedback controller which 
stabilizes the spacecraft to a desired reference position. The outer-most loop contains the ECG, which adjusts the reference to the closed-loop system in order to enforce state and control constraints. The controller takes the following form,

$$
\delta u(t)=K_{L Q} \delta \xi(t)+\Gamma v(t)-\hat{w}(t),
$$

where $K_{L Q}$ is a stabilizing feedback gain, $\Gamma$ is defined as,

$$
\Gamma=\left(-\left[I_{3} 0\right]\left(A_{l}+B_{c} K_{L Q}\right)^{-1} B\right)^{-1},
$$

and where $v(t)$ is the commanded reference from the ECG.

The ECG modifies the commanded reference $v(t)$ to the LQR so that constraints are enforced. The ECG is a discrete-time predictive scheme that updates the commanded reference every $t_{k}=k \Delta T$ seconds with $v(t)=v_{0 \mid k}$ for $t_{k} \leq t<t_{k+1}$. The discrete-time prediction model is based on (14) with control input converted to discrete time:

$$
\delta \xi_{k+1}=A_{d} \delta \xi_{k}+B_{d} v_{k}
$$

where $A_{d}$ and $B_{d}$ are the discretized state space matrices of the closed-loop system defined as follows,

$$
A_{d}=e^{A_{l} \Delta T}
$$

and

$$
B_{d}=A_{l}^{-1}\left(e^{A_{l} \Delta T}-I_{6}\right) B_{c} .
$$

During the circumnavigation phase, the rotating hyperplane constraint in (17) and control constraint in (15) are applied. In the landing phase, the rotating hyperplane plane constraint is replaced with a landing pyramid constraint, which is detailed in (19). To enforce these constraints, the ECG uses finitely determined subsets $\tilde{O}_{\infty}$ of $O_{\infty}$, where $O_{\infty}$ is defined as the set all tuples $\left(\delta \xi_{k}, \rho_{k}, \bar{x}_{k}\right)$ such that the closed-loop response to the predicted input

$$
v_{j \mid k}=\rho_{k}+\bar{C} \bar{x}_{j \mid k}, j=0,1, \cdots
$$

satisfies constraints for all time. The variables $\rho_{k} \in \mathbb{R}^{3}$ and $\bar{x}_{j \mid k} \in \mathbb{R}^{\bar{n}}, \bar{n} \geq 0$, are auxiliary states and evolve over a semi-infinite prediction horizon according to

$$
\begin{gathered}
\bar{x}_{j+1 \mid k}=\bar{A} \bar{x}_{j \mid k}, j \geq 0, \bar{x}_{0 \mid k}=\bar{x}_{k}, \\
\rho_{j \mid k}=\rho_{k}, j \geq 0,
\end{gathered}
$$

where $\bar{A}$ is Schur. The ECG determines $\rho_{k}$ and $\bar{x}_{k}$ on-board as the solution to the following quadratic program:

$$
\begin{array}{ll}
\operatorname{minimize} & \left\|\rho_{k}-r\right\|\left\|_{S_{1}}^{2}+\right\| \bar{x}_{k} \|_{S_{2}}^{2}, \\
\text { subject to } & \left(\delta \xi_{k}, \rho_{k}, \bar{x}_{k}\right) \in \tilde{O}_{\infty},
\end{array}
$$

where $r$ is the desired reference, $S_{1} \in \mathcal{S}_{+}, S_{2} \in \mathcal{S}_{+}$are positive definite matrices, and the pair $\left(S_{2}, \bar{A}\right)$ satisfies the Lyapunov equation. In the case where the constraints are linear, computational procedures exist to determine polyhedral $\tilde{O}_{\infty}$, such as in [21], which involve stacking up constraints corresponding to the predicted response over a sufficiently long but finite horizon, tightening constraints slightly in steadystate and eliminating redundant constraints (if computations of $\tilde{O}_{\infty}$ are off-board or sufficient on-board computing power is available). See [22].

There exist a few options for $\bar{A}$ and $\bar{C}$ in (34) and (35). This paper makes us of shift sequences, as done in [23] with,

$$
\bar{A}=\left[\begin{array}{ccccc}
0 & I_{3} & 0 & 0 & \ldots \\
0 & 0 & I_{3} & 0 & \ldots \\
0 & 0 & 0 & I_{3} & \ldots \\
0 & 0 & 0 & 0 & \ldots \\
\vdots & \vdots & \vdots & \vdots & \ddots
\end{array}\right]
$$

and

$$
\bar{C}=\left[\begin{array}{lllll}
I_{3} & 0 & 0 & 0 & \ldots
\end{array}\right] \text {. }
$$




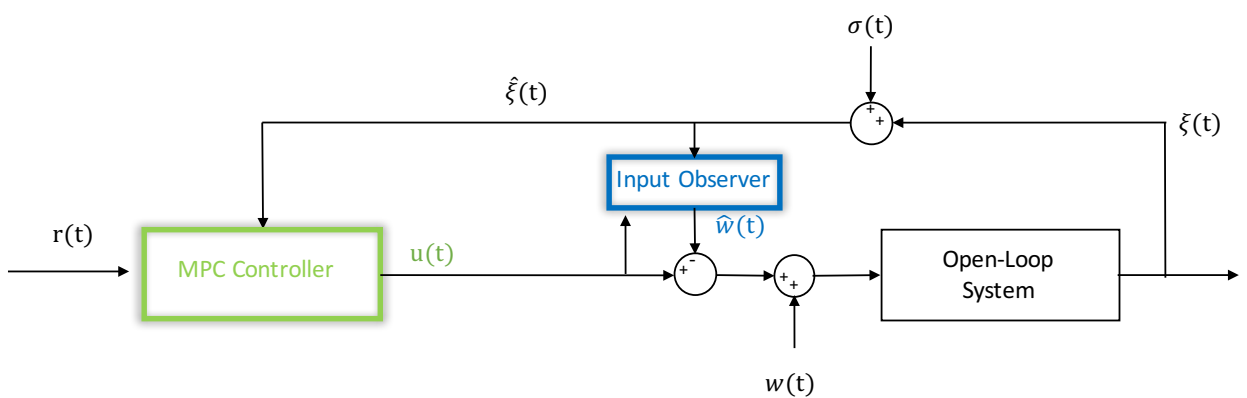

Figure 5: The MPC control strategy

\section{Nonlinear Model Predictive Control}

The nonlinear MPC approach is a discrete-time control strategy that computes the control $u_{0 \mid k}=u\left(t_{k}\right)$ to be applied at time $t_{k}=k \Delta T$, where $\Delta T$ is the sampling period of the MPC controller, as the solution to a nonlinear programming problem. Define the primal optimization variable

$$
z_{k}=\left[\begin{array}{lllllll}
u_{0 \mid k}^{T} & \xi_{1 \mid k}^{T} & u_{1 \mid k}^{T} & \cdots & \xi_{N-1 \mid k} & u_{N-1 \mid k} & \xi_{N \mid k}
\end{array}\right]^{T},
$$

and the cost function

$$
J\left(z_{k}, r_{k}\right)=\left\|\xi_{N \mid k}-r_{k}\right\|_{P}^{2}+\sum_{i=0}^{N-1}\left\|\xi_{i \mid k}-r_{k}\right\|_{Q}^{2}+\left\|u_{i \mid k}\right\|_{R}^{2},
$$

where $(Q, R) \in \mathcal{S}_{+}$are weighting matrices and $r_{k}$ is the reference. The nonlinear program may then be written as follows

$$
\begin{gathered}
\min _{z_{k}} \quad J\left(z_{k}, r_{k}\right) \\
\text { subject to } \quad \xi_{i \mid k}-f_{d}\left(\xi_{i-1 \mid k}, u_{i-1 \mid k}\right)=0, \quad i=1,2, \cdots, N \\
c\left(\xi_{i \mid k}\right) \leq 0, \quad i=1,2, \cdots, N \\
u_{\min } \leq u_{i \mid k} \leq u_{\max }, \quad i=1,2, \cdots, N-1, \\
\xi_{0 \mid k}=\hat{\xi}_{k}
\end{gathered}
$$

where $f_{d}$ is the Euler discretization of (13) and represents the nonlinear dynamics of the system with estimated gravity forces. During the circumnavigation phase $c=c_{s e}$ where $c_{s e}$ is the safety ellipsoid constraint (16). After transitioning to the navigation phase the constraint changes to $c=\left[\begin{array}{ll}c_{l p} & c_{n o}\end{array}\right]^{T}$ where $c_{l p}$ is the landing paraboloid defined in (18) and $c_{n o}$ is the no overshoot plane constraint (17). The output of the NMPC controller is then combined with the input observer to produce the final control $u\left(t_{k}\right)=u_{0 \mid k}-\hat{w}\left(t_{k}\right)$; the MPC control loop is shown in Figure 5.

We use a specialized nonlinear primal dual filter-linesearch interior point solver along the lines of IPOPT [24] which effectively and efficiently handles constrained problems. The computational burden of the algorithm is reduced by warm starting the optimization problem with previous solutions to (41). Nonconvexity during the optimization process is handled via a diagonal Hessian modification to ensure convergence to a local minimum and the linear solution to the Newton step systems are computed using MA57 [25] invoked via the MATLAB $l d l$ command.

\section{Convex Model Predictive Control}

The convex MPC approach computes the control to be applied at time $t_{k}=k \Delta T$ as the solution to a suitably defined convex programming problem. The positive definite weighting matrices $(Q, R$, and $P)$ are used to 
tune the controller. The optimization problem to be solved onboard for the control $u_{0 \mid k}$ is of the form

$$
\begin{gathered}
\min _{z_{k}} \quad J\left(z_{k}, r_{k}\right) \\
\text { subject to } \quad \delta \xi_{i \mid k}-f_{d}\left(\hat{\xi}_{k}\right)-A_{d} \delta \xi_{i-1 \mid k}-B_{d} u_{i-1 \mid k}=0, \\
i=1,2, \cdots, N \\
c\left(\xi_{i \mid k}\right) \leq 0, \quad i=1,2, \cdots, N \\
u_{\min } \leq u_{i \mid k} \leq u_{\max }, \quad i=1,2, \cdots, N-1, \\
\xi_{0 \mid k}=\hat{\xi}_{k}
\end{gathered}
$$

where $J\left(z_{k}, r_{k}\right)$ is given by $(40)$ and the discrete time state space matrices $\left(A_{d}, B_{d}\right)$ are generated by discretizing the linear equations of motion (14) which are evaluated at the current measured state and zero control i.e., $\xi_{0}=\hat{\xi}\left(t_{k}\right), u_{0}=0$. Note that the nonlinear prediction model (13) is linear in $u$ thus the choice of $u_{0}=0$ is exact. The inequality constraint function $c\left(\xi_{i \mid k}\right)$ is the rotating hyperplane constraint (17) during the circumnavigation phase and the concatenation of the landing paraboloid (18) and no overshoot constraint (20) during the landing phase. The controller architecture is the same as in the NMPC case and is shown in Figure 5.

The CMPC problem is a convex quadratic problem during the circumnavigation phase because the equality constraints are linear and the hyperplane inequality constraint is affine, allowing us to apply the powerful convex optimization tool CVX [26]. During the landing phase the problem with nonlinear inequality constraints is no longer a quadratic program but it remains a convex problem as the intersection of the convex landing parabola constraint $c_{i c}\left(x_{i}\right)<0$, the affine saturation constraint $u_{\min } \leq u_{i} \leq u_{\max }$, and the affine no overshoot plane define a convex set.

\section{E. Input Constraint Tightening}

The input observer is used to counteract the model disturbances, as in Section A. However, the input observer modifies the control output without the knowledge of the controller which can cause the total control command to violate the control constraints. To address this the control constraints are tightened by using

$$
\begin{aligned}
& u_{\max }\left(t_{k+1}\right)=u_{\max }(0)-\left\|\hat{w}\left(t_{k+1}\right)\right\|_{\infty} \\
& u_{\min }\left(t_{k+1}\right)=u_{\min }(0)+\left\|\hat{w}\left(t_{k+1}\right)\right\|_{\infty},
\end{aligned}
$$

where $\hat{w}\left(t_{k+1}\right)$ denotes the predicted disturbance at the time $t_{k+1}$ based on extrapolating the output of the input observer.

\section{Simulation Results}

In this section we present results for simulated landings on the asteroid 433 Eros using all three control schemes presented in this paper. All simulations were done using the known gravity model from Yeomans et al. [13]. Outside perturbations are assumed to be negligible and are not simulated. The parameters of the simulation are given in Table 1.

\section{A. Landing Points and Simulation Parameters}

Two landing points were chosen for the simulations. The target point shown in Figure $6 \mathrm{~b}$ is referred to as the valley landing point and is located in the deepest valley on Eros. The target point in Figure 6a is referred to as the plain landing point and is located in a broad depression in the asteroid. These points were chosen as they are in depressions in the asteroid that present a challenge and require the landing constraint as described in Section III. Note that the valley landing point is within the constant density ellipsoid used to construct the approximate gravity model (13), leading to significant model mismatch, and is thus considered more difficult than the plain landing site. Table 1 contains all the adjustable parameters used in the simulation. 


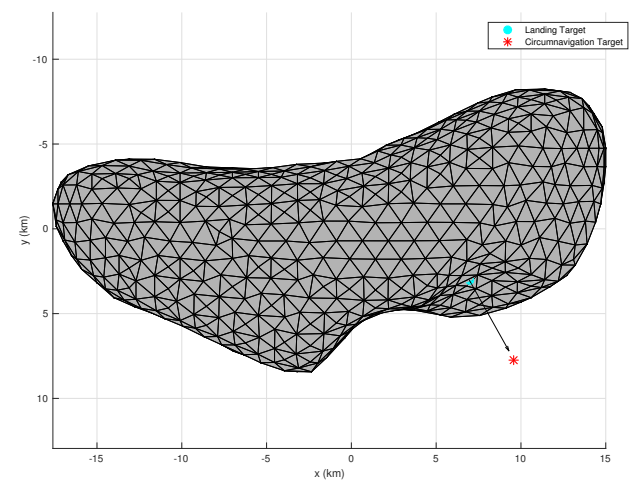

(a) The plain landing point.

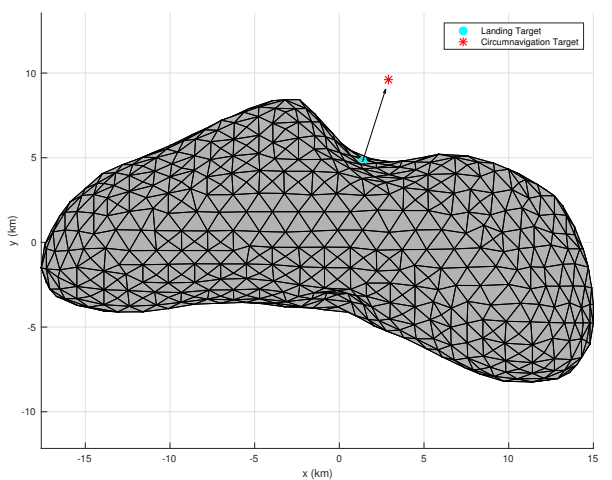

(b) The valley landing point.

Figure 6: Landing Points

Table 1: Simulation Parameters

\begin{tabular}{cccccc}
\hline \hline Parameter & Units & Value & Parameter & Units & Value \\
\hline$\mu$ & $\mathrm{m}^{3} / \mathrm{sec}^{2}$ & $4.46 \mathrm{e}-4$ & $a_{\text {safe }}$ & $\mathrm{km}$ & 22 \\
$n$ & $\mathrm{rads} / \mathrm{sec}$ & $3.3118 \mathrm{e}-4$ & $b_{\text {safe }}$ & $\mathrm{km}$ & 10 \\
$R_{0}$ & $\mathrm{~km}$ & 20 & $c_{\text {safe }}$ & $\mathrm{km}$ & 10 \\
$a$ & $\mathrm{~km}$ & 20 & $\gamma$ & - & 0.025 \\
$b$ & $\mathrm{~km}$ & 5 & $n_{\text {hyper plane }}$ & $\mathrm{deg} / \mathrm{sec}$ & 2 \\
$c$ & $\mathrm{~km}$ & 5 & $n$ & $\mathrm{rads} / \mathrm{sec}$ & $3.3118 \mathrm{e}-4$ \\
$\Delta T_{n m p c}$ & $\mathrm{sec}$ & 0.4 & $\Delta T_{\text {ecg }}$ & $\mathrm{sec}$ & 1 \\
$u_{\text {max }}$ & $\mathrm{m} / \mathrm{s}^{2}$ & 25 & $N_{\text {nmpc }}$ & - & 70 \\
$u_{\text {min }}$ & $\mathrm{m} / \mathrm{s}^{2}$ & -25 & $d_{n}$ & $\mathrm{~m}$ & 3 \\
$\eta$ & - & 0.1 & surface offset & $\mathrm{m}$ & 5 \\
$Q_{n m p c}$ & - & $10^{-3} \cdot\left(I_{3} \oplus 50 I_{3}\right)$ & $Q_{E C G}$ & - & $10^{-3} \cdot\left(I_{3} \oplus 50 I_{3}\right)$ \\
$R_{n m p c}$ & - & $I_{3}$ & $R_{E C G}$ & - & $25 I_{3}$ \\
$d_{p}$ & $\mathrm{~m}$ & 150 & $a_{p}$ & $\mathrm{~km}$ & 0.4 \\
$d_{\text {switch }}$ & $\mathrm{m}$ & 10 & $N_{E C G}$ & - & 25 \\
$Q_{c m p c}$ & - & $10^{-3} \cdot\left(I_{3} \bigoplus 75 I_{3}\right)$ & $R_{c m p c}$ & - & $I_{3}$ \\
$\Delta T_{c m p c}$ & $\mathrm{~s}$ & 0.5 & $N_{c m p c}$ & - & 50 \\
\hline \hline
\end{tabular}

\section{B. Metrics}

The three controllers are compared using four different metrics in order to evaluate fuel use, precision, constraint handling and tracking.

Fuel use: Fuel use is assumed to be proportional to the delta $v$ which is calculated as

$$
\Delta V=\int_{0}^{t_{f}}\|u(t)\|_{1} d t
$$

where $t_{f}$ is the final time. The assumption of three orthogonal sets of thrusters given in Section II leads to the use of the 1-norm in (54). 
Precision: Precision is measured using the final error which is simply the 2-norm distance to the landing point at the end of the simulation

$$
\Delta r_{f}=\left\|\mathbf{r}\left(t_{f}\right)-\mathbf{r}_{L}\right\|_{2} .
$$

Constraint Violation: The constraint violation is integrated to provide a measurement of constraint satisfaction

$$
c_{v}=\int_{0}^{t_{f}} \sum_{i} \max \left(C_{i}(\xi, u), 0\right) d t
$$

Note that the sum is taken over the components of the vector

$$
C=\left[\begin{array}{c}
u(t)-u_{\max } \\
-u(t)+u_{\min } \\
c_{\text {state }}
\end{array}\right],
$$

and $c_{\text {state }}$ either corresponds to the safety ellipsoid constraint (16) or to the landing paraboloid and the no overshoot hyperplane (18), (20) depending on the phase.

Tracking Error: The root mean square error (RMSE) is used to quantify the tracking error

$$
e_{r m s}=\sqrt{\frac{1}{t_{e}} \int_{0}^{t_{e}}\left\|\xi(t)-\xi_{e}\right\|_{2}^{2} d t+\frac{1}{t_{f}-t_{e}} \int_{t_{e}}^{t_{f}}\left\|\xi(t)-\xi_{L}\right\|_{2}^{2} d t},
$$

where $t_{e}$ and $\xi_{e}$ are the circumnavigation time and target and $\xi_{L}$ is the landing target.

\section{Controller Comparison}

For both target points, the controllers were able to successfully avoid constraint violation and reach the landing zones, as shown in Figures 7 through 10. Figures 11 through 16 show the time histories of the positions relative to the asteroid, the control applied with limits displayed, velocity, and the disturbance estimate which accounts for the model error. In almost all cases a steady state is reached as the spacecraft approaches the target point. The exception is the CMPC controller landing in the valley (Figure 15) where interactions between the input observer and the controller lead to repeated collisions with the constraints.

Overall the ECG used less fuel during the circumnavigation phase while the NMPC controller offers superior constraint handling and disturbance rejection during the landing phase. The reference shifting by the ECG leads to smaller errors, less aggressive control action, and lower fuel use. This behaviour is due to the rotating hyperplane constraint, which forces the ECG to modify the reference gradually as the hyperplane shifts around the asteroid. However, the ECG updates the reference at a slow rate to accommodate long prediction horizons. This, in combination with the use of lower fidelity models for both the dynamics and the constraints, leads to more constraint violations. This effect is more noticeable during the landing phase where un-modelled gravitational disturbance forces are more prevalent.

In contrast, the MPC controllers proved to have superior constraint handling and disturbance rejection performance. The relatively fast sampling period and the increased model accuracy due to the use of more accurate models lead to superior constraint handling, especially during the landing phase where disturbance forces are more prevalent. However, during the circumnavigation phase, the finite horizon nature of MPC controllers causes the spacecraft to approach the safety ellipsoid constraint aggressively as, far away from the asteroid, the controller does not perceive the constraint. By the time the constraint is perceptible to the controller the spacecraft is moving at high speeds and, due to limited actuator authority, the controller overshoots the ellipsoid; this is visible in Figure 8. This issue can be resolved without increasing the horizon length $N$, and thus computational complexity, by increasing the sampling period. However, during the landing phase, the shorter sampling periods are necessary to improve disturbance rejection.

The controllers were tuned separately but a single set of weighting matrices was used by each for both circumnavigation and landing. The NMPC and CMPC approaches proved difficult to tune. The scale differences of the errors at different points along the trajectory, especially between the circumnavigation and landing phases, made finding $Q, R$, and $P$ matrices that performed well during both phases challenging. Tunings that provide an ideal critically damped response during the critical landing phase cause aggressive, inefficient behaviour during the circumnavigation phase; this is visible in Figures 11 and 12. 


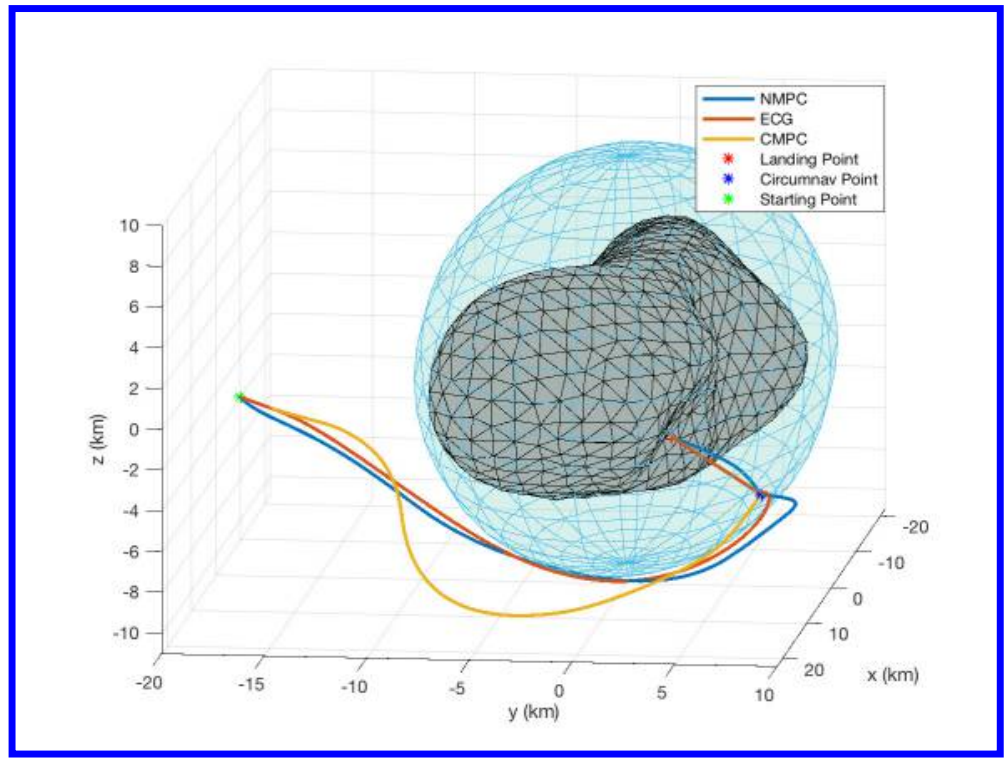

Figure 7: The trajectories for the circumnavigation portion of the plain landing scenario.

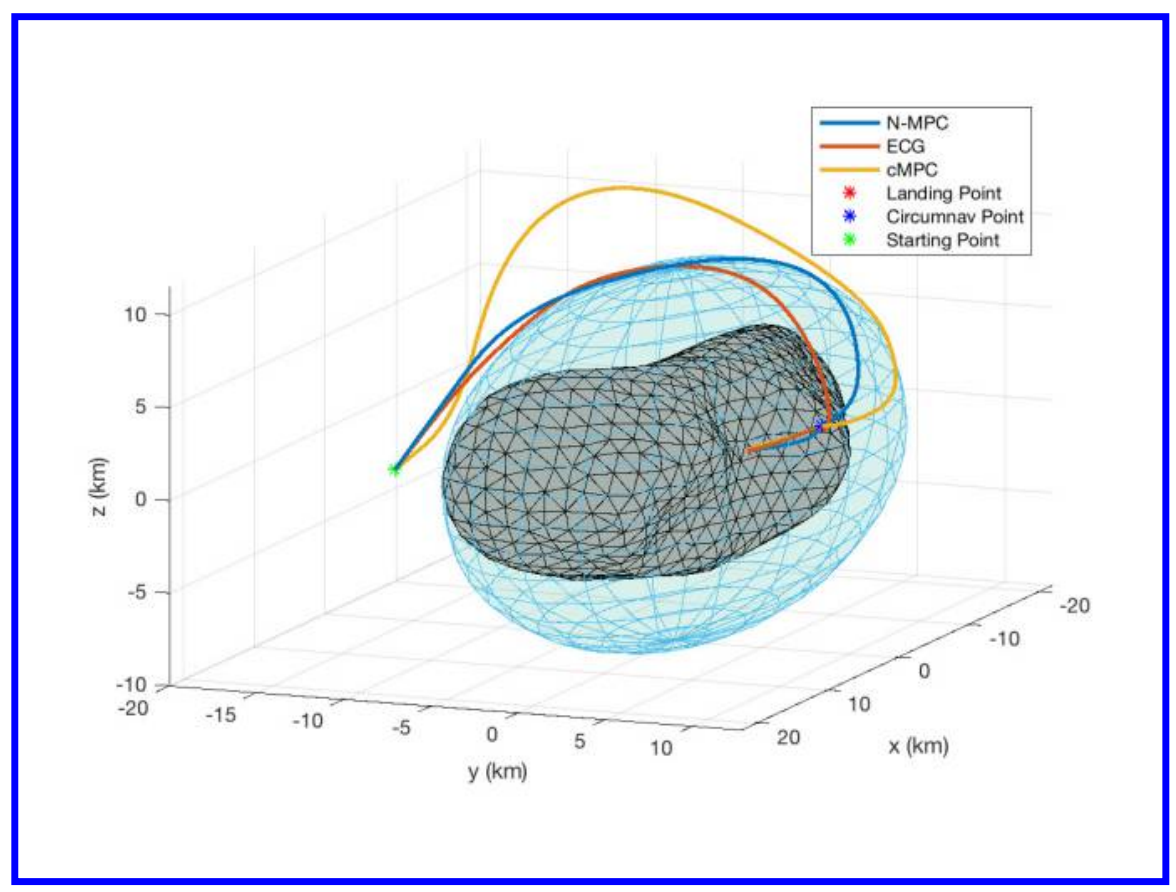

Figure 8: The trajectories for the circumnavigation portion of the valley landing scenario. 


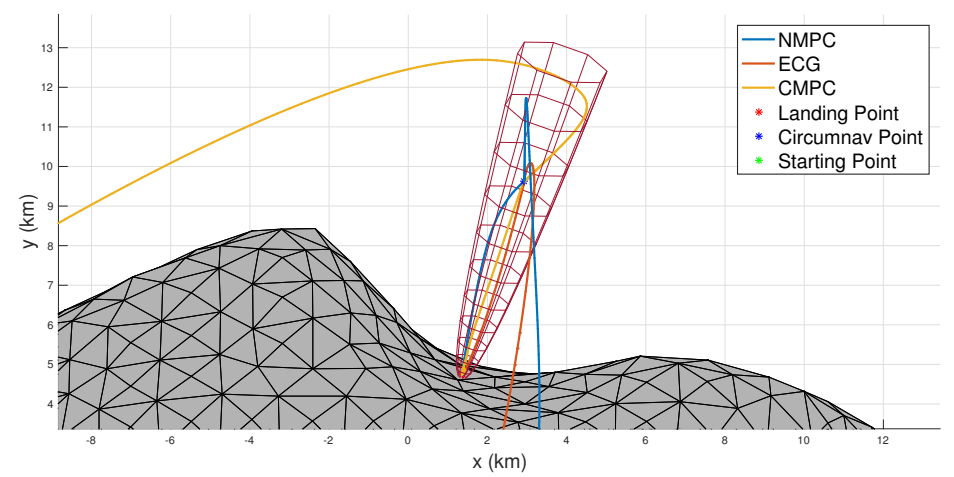

Figure 9: A close-up of the landing portion of the valley landing scenario

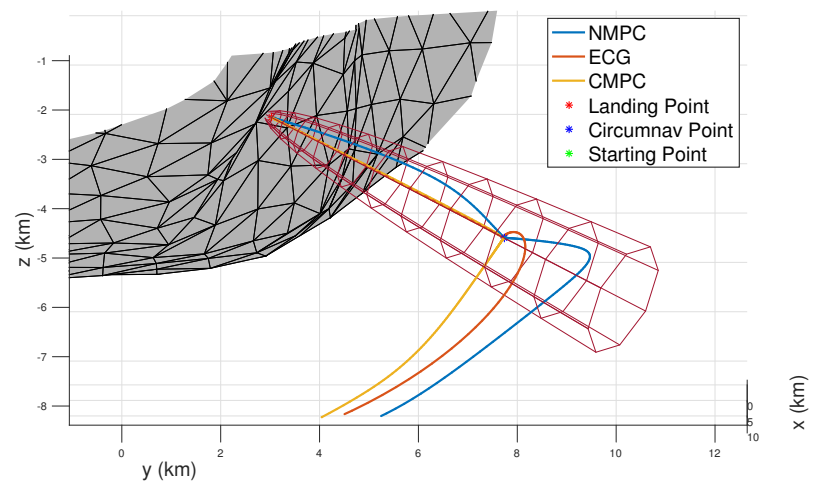

Figure 10: A close-up of the landing portion of the plain landing scenario
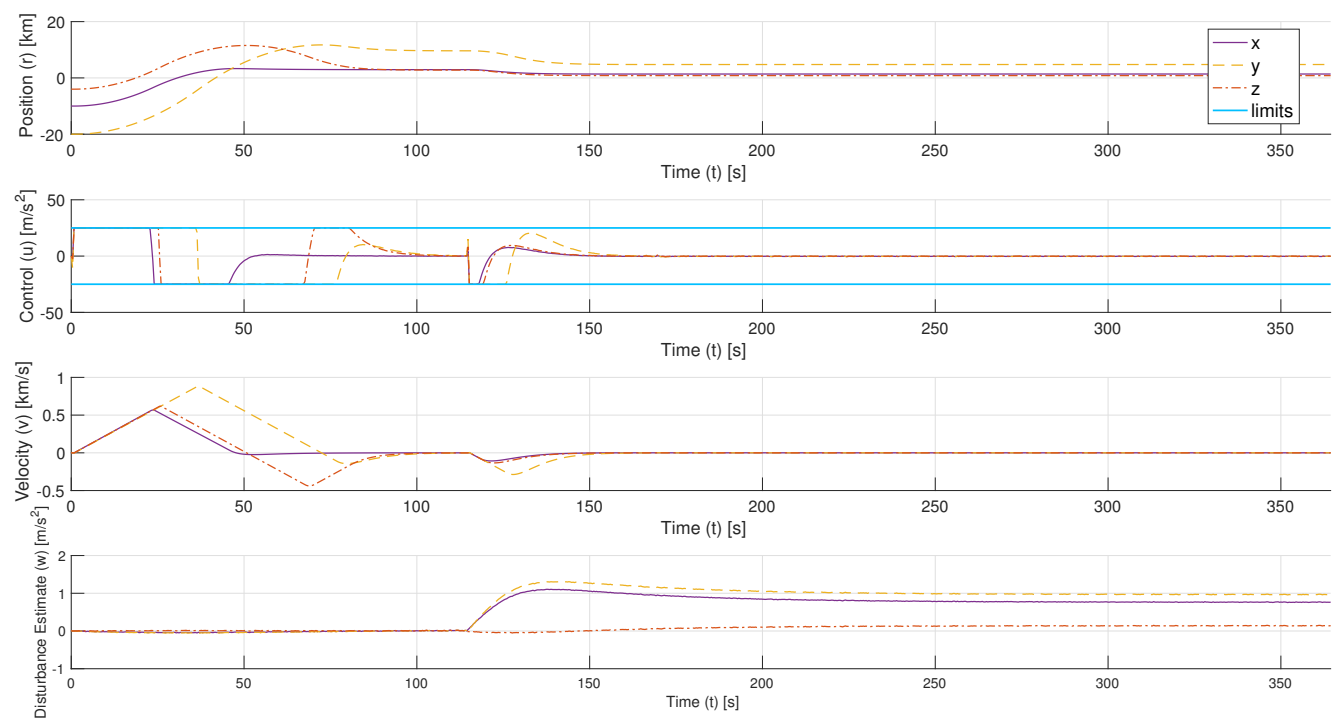

Figure 11: Simulation results, Valley, NMPC 

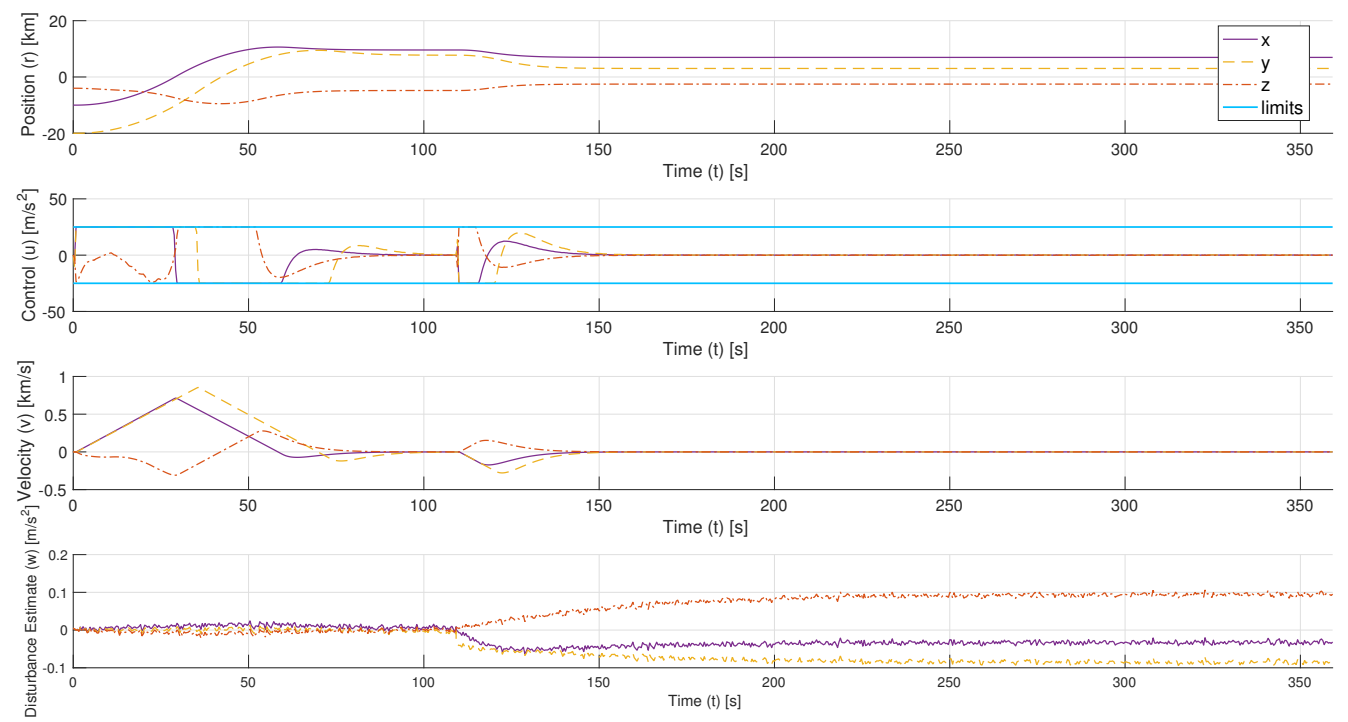

Figure 12: Simulation results, Plain, NMPC
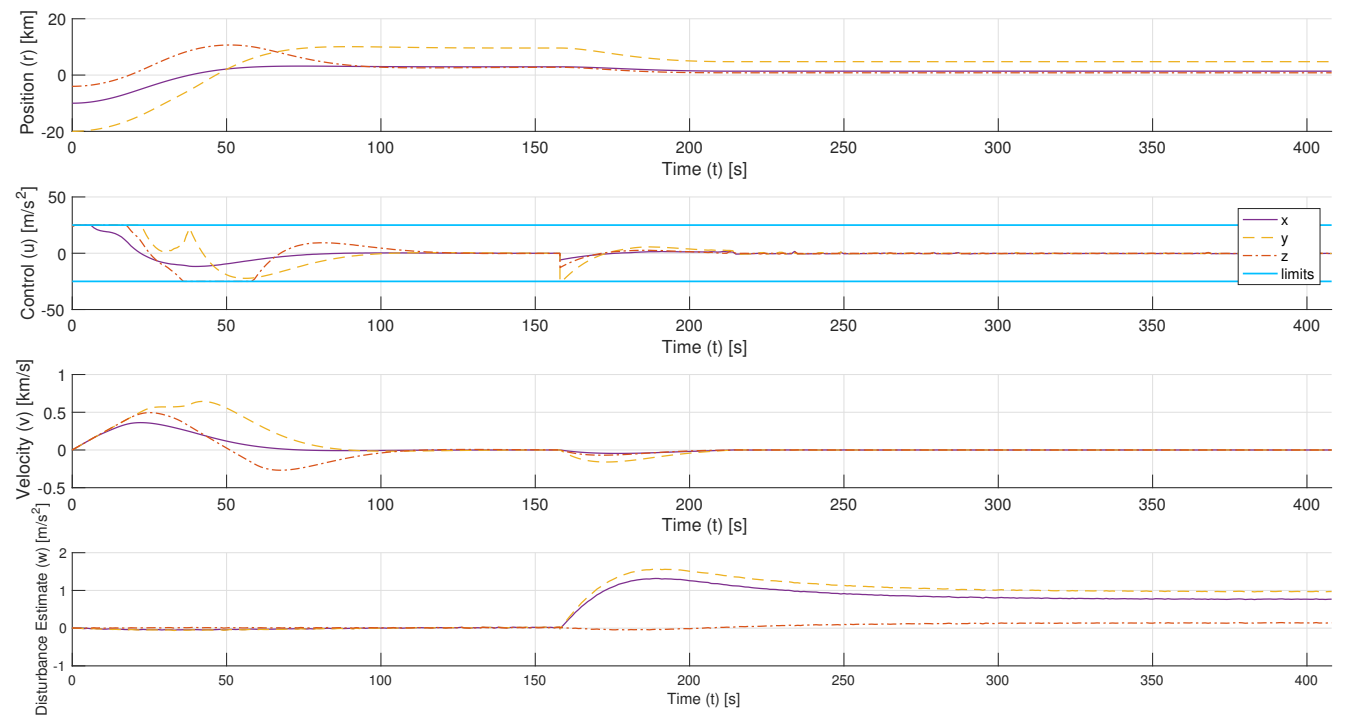

Figure 13: Simulation results, Valley, ECG

15 of 21 

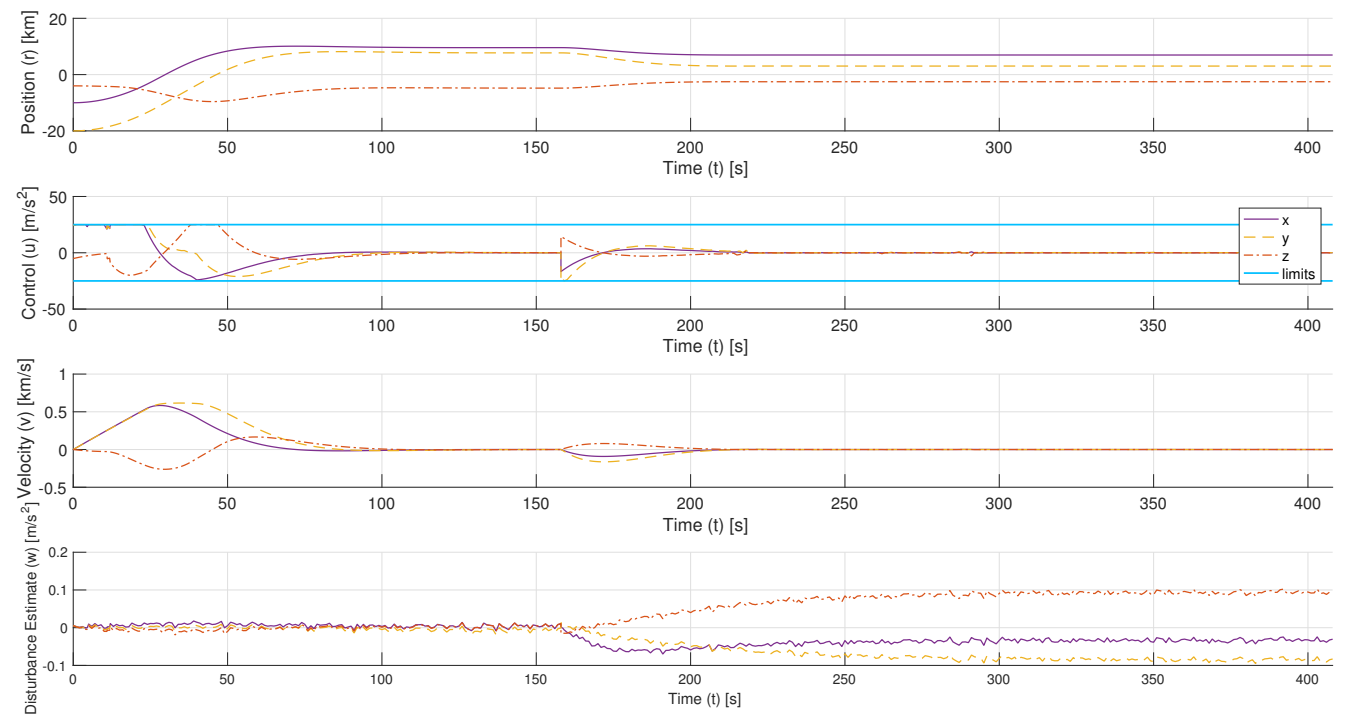

Figure 14: Simulation results, Plain, ECG
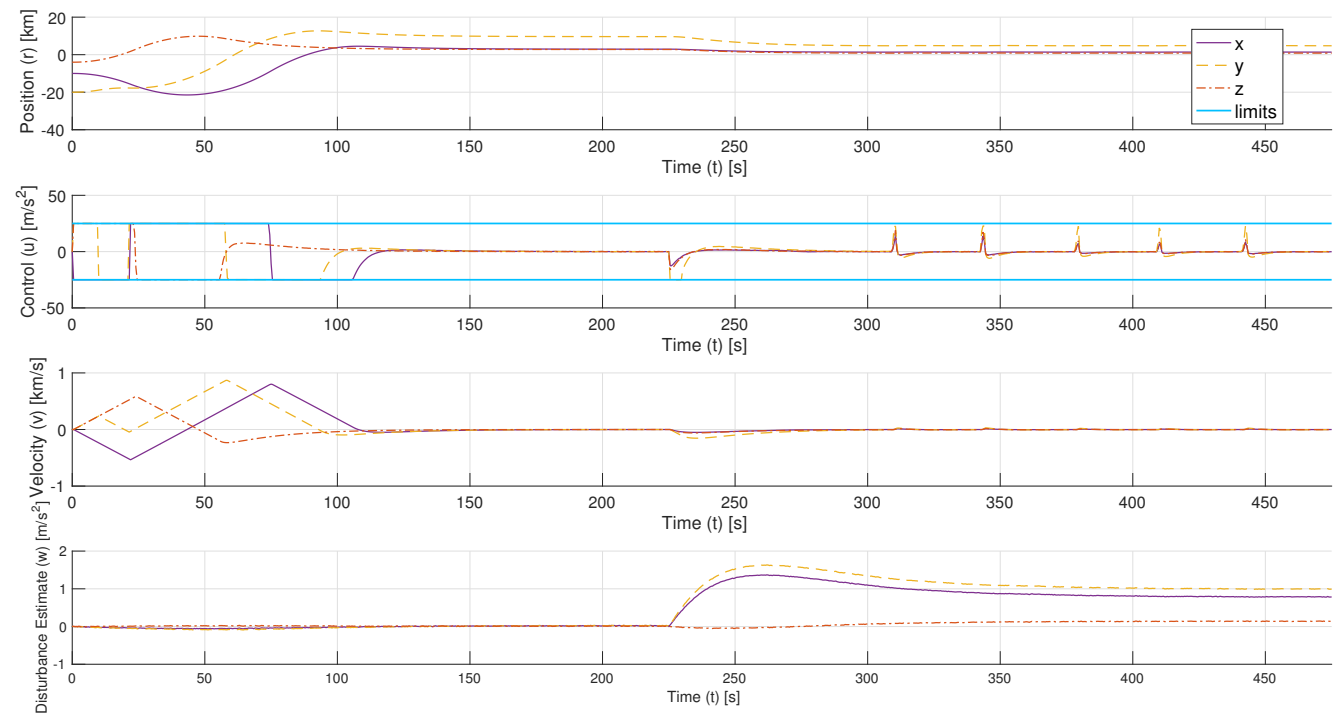

Figure 15: Simulation results, Valley, CMPC

16 of 21 

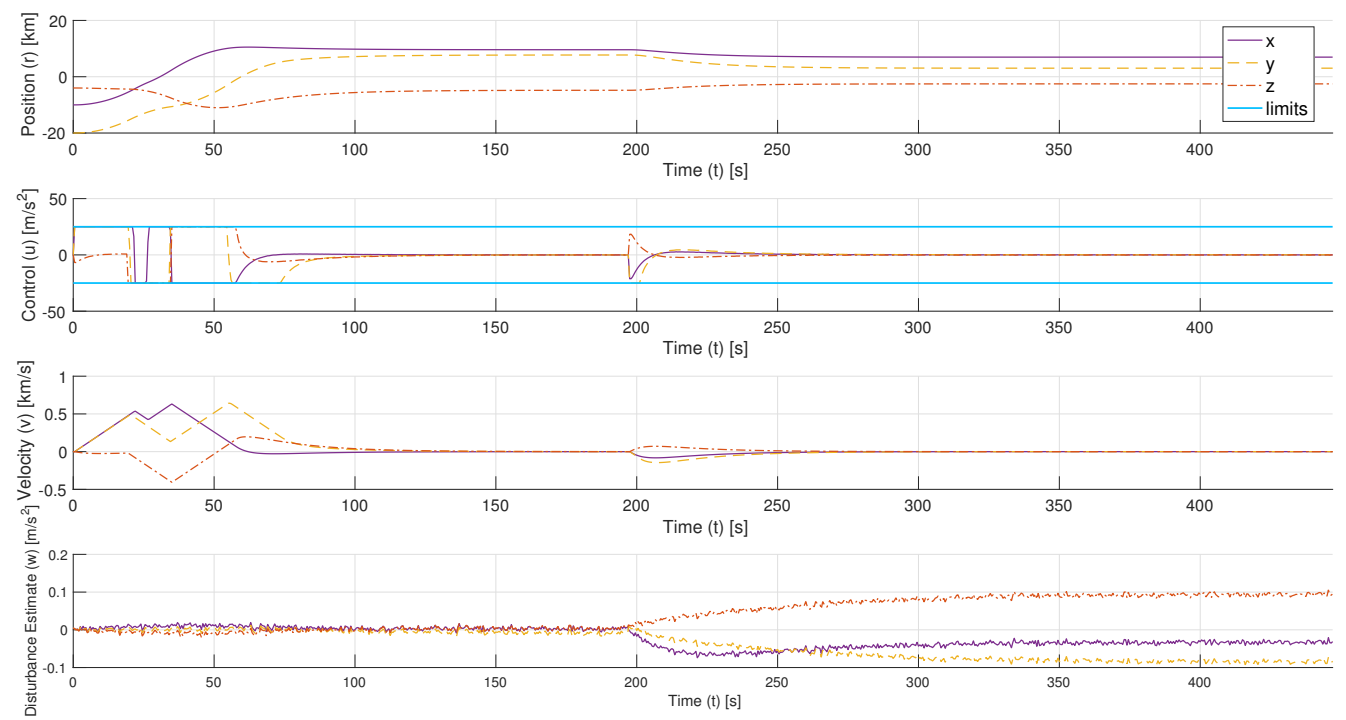

Figure 16: Simulation results, Plain, CMPC

The metrics for each case and each controller are given in Tables 2 through 4 . The ECG provided the best overall fuel economy while the NMPC had the best steady state tracking and proved to be the most robust to estimation error and disturbances. Both had comparable overall tracking and error handling results. The CMPC performed better than the NMPC in the plain case but was less robust overall.

Table 2: ECG Results

\begin{tabular}{ccccc}
\hline \hline Metric & $\Delta V[\mathrm{~km} / \mathrm{s}]$ & $\Delta r_{f}[\mathrm{~m}]$ & $e_{r m s}$ & $c_{v}$ \\
\hline Valley & 4.49 & 3.20 & 14.1 & $1.90 \times 10^{-3}$ \\
Plain & 4.23 & 4.20 & 13.9 & $2.45 \times 10^{-4}$ \\
\hline \hline
\end{tabular}

Table 3: NMPC Results

\begin{tabular}{ccccc}
\hline \hline Metric & $\Delta V[\mathrm{~km} / \mathrm{s}]$ & $\Delta r_{f}[\mathrm{~m}]$ & $e_{r m s}$ & $c_{v}$ \\
\hline Valley & 6.56 & 2.48 & 16.20 & $1.59 \times 10^{-3}$ \\
Plain & 6.00 & 0.45 & 16.45 & $1.24 \times 10^{-3}$ \\
\hline \hline
\end{tabular}

Table 4: CMPC Results

\begin{tabular}{lcccc}
\hline \hline Metric & $\Delta V[\mathrm{~km} / \mathrm{s}]$ & $\Delta r_{f}[\mathrm{~m}]$ & $e_{r m s}$ & $c_{v}$ \\
\hline Valley & 7.96 & 25.14 & 17.65 & $4.3 \times 10^{-2}$ \\
Plain & 5.39 & 13.44 & 13.46 & $7.5 \times 10^{-5}$ \\
\hline \hline
\end{tabular}

\section{Interaction between MPC controllers and the input observer}

In this section we investigate the interactions between the input observer and the MPC controllers. A similar study was performed in reference [9] for the ECG control strategy. The MPC formulations used in this paper do not provide zero offset tracking error in the presence of DC gain mismatch between the prediction model 
and the real system. As a result final position errors for the NMPC controller without the input observer in Table 5 is an order of magnitude worse than the final error with the input observer (Table 3). Aggressive values of $\gamma$ the observer gain can be shown to improve the final position error but they can cause constraint violations during transients and can, in some cases, destabilize the controller. These negative interactions between the controllers and the input observer proved to be the limiting factor in the achievable targeting precision. This is visible in Table 5 where the NMPC controller alone perfectly satisfies constraints while the NMPC controller in combination with the input observer violates control constraints.

The interactions are even more pronounced in the CMPC case. The input observer coupled with model mismatch causes the spacecraft to repeatedly bounce off the no overshoot constraint, leading to the spiking visible in Figure 15. In the plain case, the use of the input observer degraded controller performance across all metrics while in the valley case it reduces fuel consumption while degrading all other metrics.

In summary, an input observer or another alternate form of integral action or adaptation, is necessary to achieve precise positioning. Unfortunately most of these strategies degrade transient performance and can cause constraint violation. These effects can be partially mitigated through careful tuning of the interactions between the input observer and the controller. However, a more robust methodology is desirable for autonomous operation. Overall the NMPC controller, correctly tuned, was robust enough to reject the transient effects of the input observer and achieve steady state error of roughly the same order of magnitude as the state estimation error. In contrast we were unable to resolve the negative interactions between the CMPC controller and the input observer through tuning.

Table 5: NMPC results without input observer

\begin{tabular}{ccccc}
\hline \hline Metric & $\Delta V[\mathrm{~km} / \mathrm{s}]$ & $\Delta r_{f}[\mathrm{~m}]$ & $e_{r m s}$ & $c_{v}$ \\
\hline Valley & 6.55 & 42.67 & 16.20 & 0 \\
Plain & 6.02 & 3.65 & 16.45 & 0 \\
\hline \hline
\end{tabular}

Table 6: CMPC results without input observer

\begin{tabular}{lcccc}
\hline \hline Metric & $\Delta V[\mathrm{~km} / \mathrm{s}]$ & $\Delta r_{f}[\mathrm{~m}]$ & $e_{r m s}$ & $c_{v}$ \\
\hline Valley & 7.44 & 63.68 & 17.34 & 0 \\
Plain & 5.397 & 6.52 & 13.42 & 0 \\
\hline \hline
\end{tabular}

\section{E. Sensitivity to initial conditions}

Simulations starting from twenty-seven initial positions in a 10x10x10 km cube centered around the point $r=\left[\begin{array}{lll}-10 & -25 & -5\end{array}\right]$ were run for each controller to test robusness to the intial position. Figures 18, 17, and 19 show the results for the NMPC controller, ECG and CMPC controllers. Each controller proved to be robust to such variances in initial conditions. We also note that for certain initial conditions, outside the 1-norm ball considered above, the trajectories may diverge, if the controller parameters are not updated. 


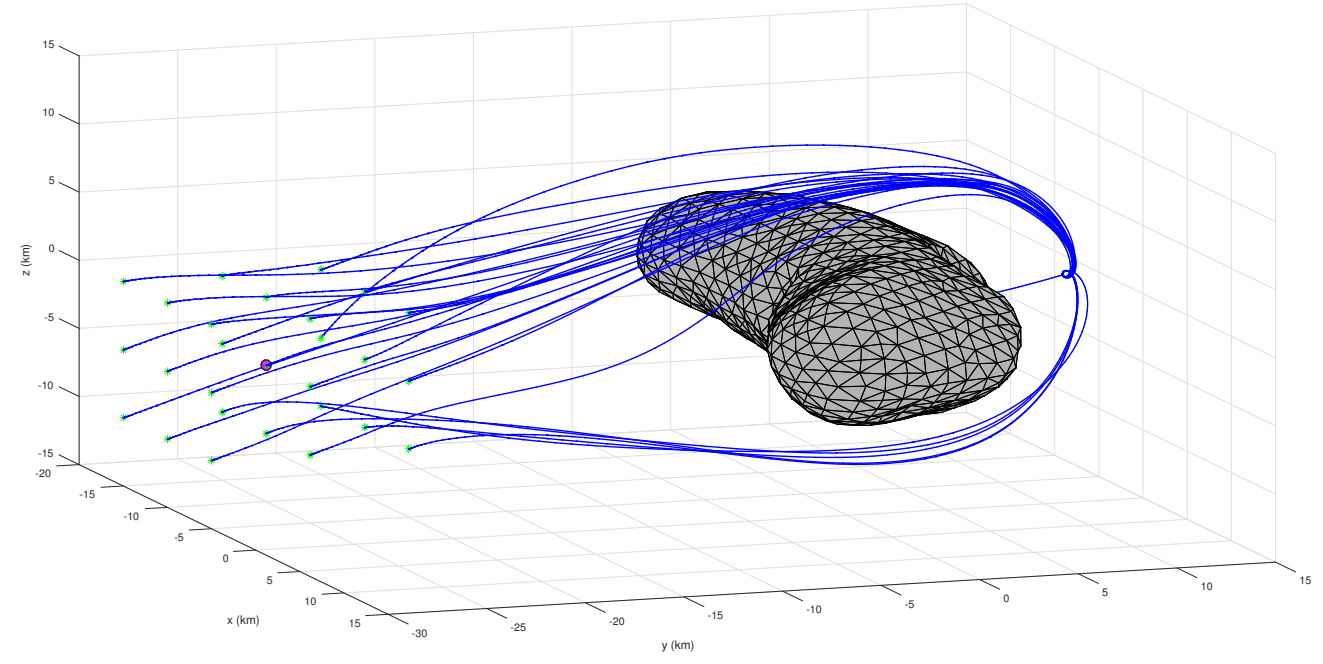

Figure 17: The results of varying the initial conditions for the ECG controller. The purple circle indicates the centre of the box.

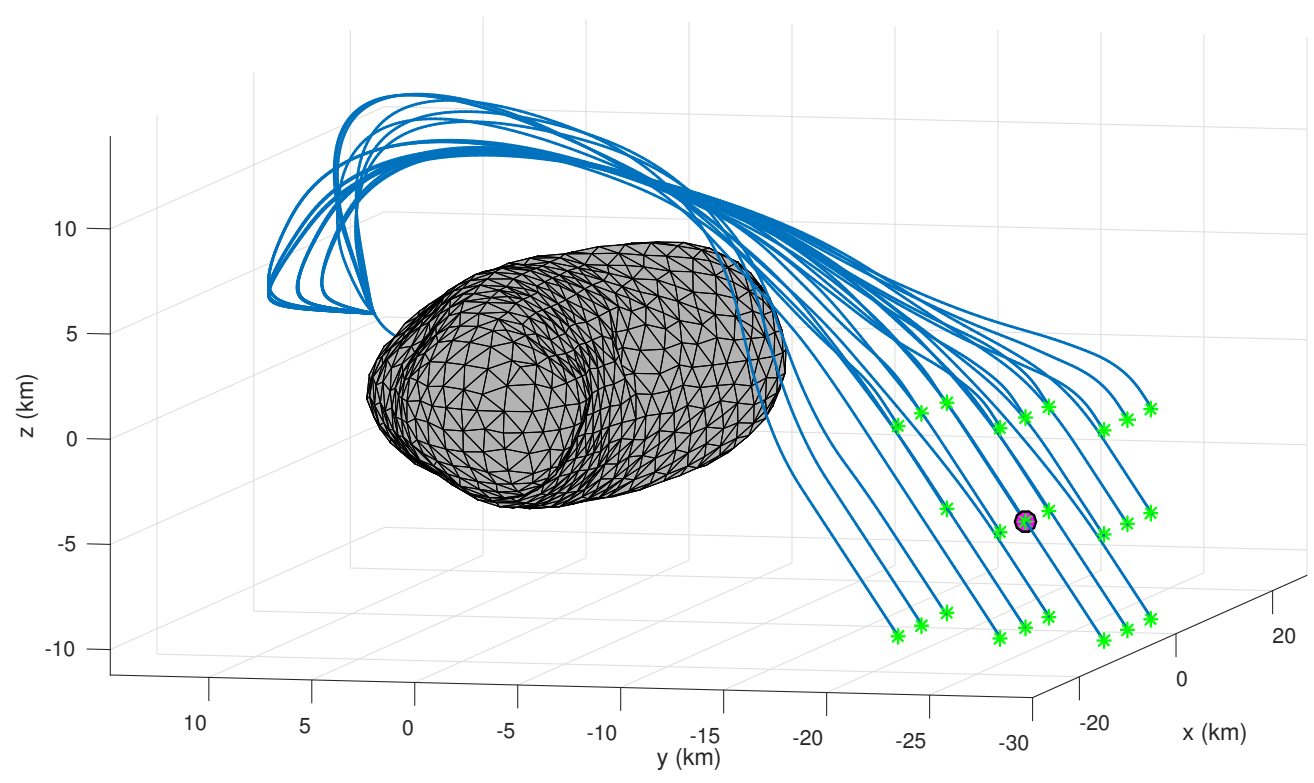

Figure 18: The results of varying the initial conditions for the NMPC controller. The purple circle indicates the centre of the box. 


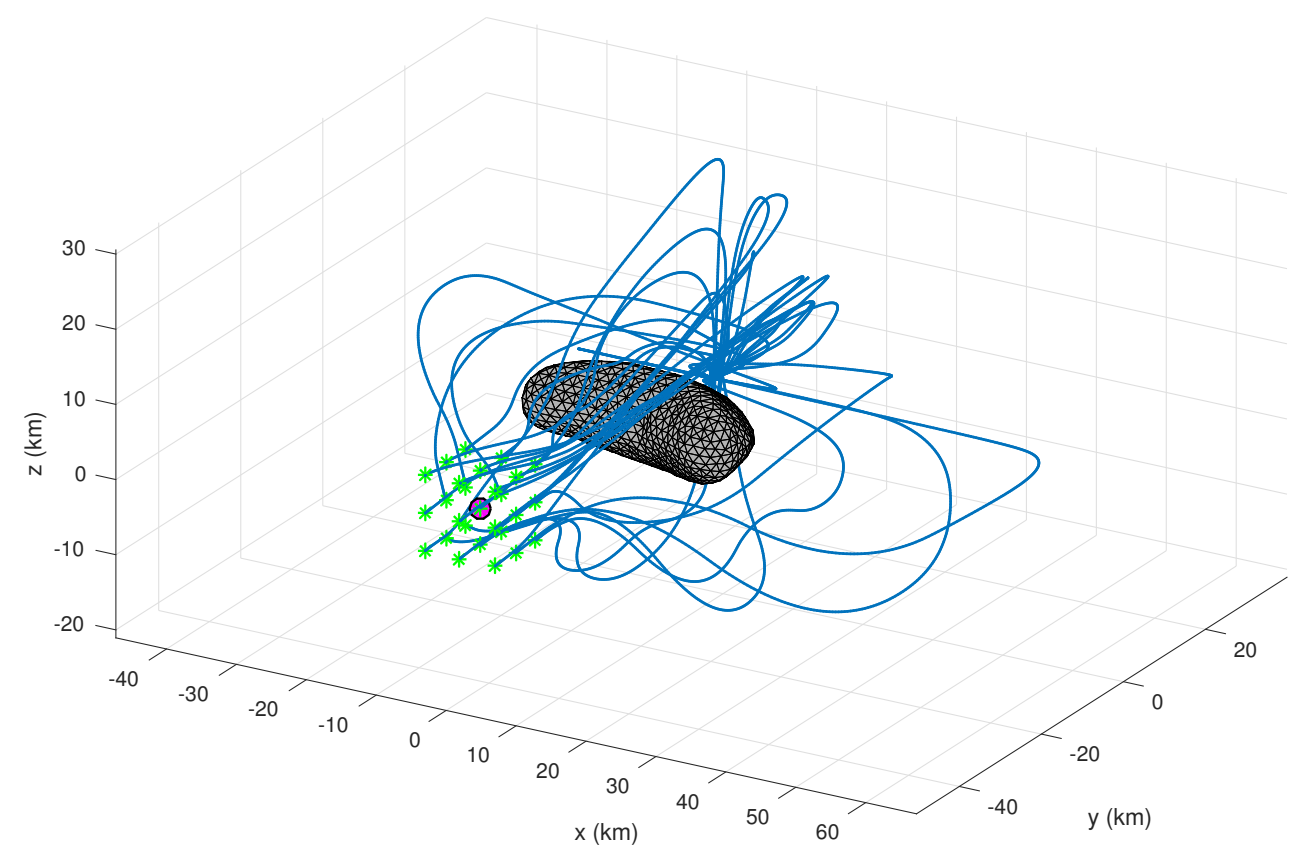

Figure 19: The results of varying the initial conditions for the CMPC controller. The purple circle indicates the centre of the box.

\section{Conclusion}

In this paper two controllers, a nonlinear model predictive controller and a convex model predictive controller, were designed for soft landing on an asteroid and compared to a previously developed solution that exploits the extended command governor. An input observer was used to compensate for model mismatch and improve positioning precision. We found that the MPC controllers have superior constraint handling performance at the expense of decreased fuel efficiency and sensitive to tuning. In particular, the interactions between the input observer and the MPC controllers were difficult to tune. An NMPC controller with a path planning algorithm layered over it, similar to the ECG appears to be a promising direction for future research. 


\section{References}

${ }^{1}$ ESA, "Celebrating a Year at the Comet," August 2015, http://www.esa.int, Retrieved September 2015.

${ }^{2}$ Brown, D. and Neal-Jones, N., "NASA Press Release 15-056 - NASA's OSIRIS-REx Mission Passes Critical Milestone," March 2015, http://www.NASA.gov/press/2015/march/nasa-s-osiris-rex-mission-passes-critical-milestone, Retrieved September 2015 .

${ }^{3}$ Wie, B., Lappas, V., and Gil-Fernández, J., "Attitude and Orbit Control Systems," The International Handbook of Space Technology, edited by M. Macdonald and V. Badescu, Springer, 2014, pp. 360-368.

${ }^{4}$ Scheeres, D. J., "Orbital Motion in Strongly Perturbed Environments: Applications to Asteroid, Comet and Planetary Satellite Orbiters (Springer Praxis Books)," 2012.

${ }^{5}$ Carson, J., Açıkmese, B., Murray, R. M., and MacMynowski, D. G., "Robust model predictive control with a safety mode: Applied to small-body proximity operations," Proceedings of the AIAA Guidance, Navigation, and Control Conference and Exhibit, 2008.

${ }^{6}$ Ruoyan, W., Xiaoqing, R., Sie, O., and Yao, X., "A Method of Guidance, Navigation, and Control for Soft Landing on Asteroid Based on Constrained MPC," Proceedings of the 11th World Congress on Intelligent Control and Automation, 2014, pp. 4213-4217.

${ }^{7}$ Lee, D., Sanyal, A. K., Butcher, E. A., and Scheeres, D. J., "Finite-time control for spacecraft body-fixed hovering over an asteroid," IEEE Transactions on Aerospace and Electronic Systems, Vol. 51, No. 1, 2015, pp. 506-520.

${ }^{8}$ Yang, H. and Baoyin, H., "Fuel-optimal control for soft landing on an irregular asteroid," IEEE Transactions on Aerospace and Electronic Systems, Vol. 51, No. 3, 2015, pp. 1688-1697.

$\checkmark{ }^{9}$ Dunham, W., Petersen, C., and Kolmanovsky, I., "Constrained control for soft landing on an asteroid with gravity model uncertainty," 2016 American Control Conference (ACC), IEEE, 2016, pp. 5842-5847.

${ }^{10}$ Petersen, C., Jaunzemis, A., Baldwin, M., Holzinger, M., and Kolmanovsky, I. V., "Model Predictive Control and extended command governor for improving robustness of relative motion guidance and control," Proceedings of AAS/AIAA Space Flight Mechanics Meeting, 2014.

${ }^{11}$ Weiss, A., Predictive, Adaptive, and Time-Varying Control of Spacecraft Orbits and Attitude, Ph.D. thesis, University of Michigan, 2013.

12 Jewison, C., Erwin, R. S., and Saenz-Otero, A., "Model Predictive Control with Ellipsoid Obstacle Constraints for Spacecraft Rendezvous," IFAC-PapersOnLine, Vol. 48, No. 9, 2015, pp. 257-262.

${ }^{13}$ Yeomans, D. K., Antreasian, P. G., Barriot, J.-P., Chesley, S. R., Dunham, D. W., Farquhar, R. W., Giorgini, J. D., Helfrich, C. E., Konopliv, A. S., McAdams, J. V., Miller, J., Jr., W. M. O., Scheeres, D. J., Thomas, P. C., Veverka, J., and Williams, B. G., "Radio Science Results During the NEAR-Shoemaker Spacecraft Rendezvous with Eros," Science, Vol. 289, 2000, pp. 2085-2088.

-14Zuber, M., Smith, D., Cheng, A., Garvin, J., Aharonson, O., Cole, T., Dunn, P., Guo, Y., Lemoine, F., Neumann, G., Rowlands, D., and Torrence, M., "The Shape of 433 Eros from the NEAR-Shoemaker Laser Rangefinder," Science, Vol. 289, 2000, pp. 2097-2101.

${ }^{15}$ Hughes, P. C., Spacecraft attitude dynamics, Courier Corporation, 2012.

$\checkmark{ }^{16}$ Kaasalainen, M., Torppa, J., and Muinonen, K., "Optimization methods for asteroid lightcurve inversion: II. The complete inverse problem," Icarus, Vol. 153, 2001, pp. 37-51.

${ }^{17}$ Montenbruck, O. and Gill, E., Satellite orbits: models, methods and applications, Springer Science \& Business Media, 2012.

${ }^{18}$ Werner, R. A., "Spherical harmonic coefficients for the potential of a constant-density polyhedron," Computers 83 Geosciences, Vol. 23, No. 10, 1997, pp. 1071-1077.

${ }^{19}$ Miller, J., Konopliv, A. S., Antreasian, P. G., Bordi, J. J., Chesley, S., Helfrich, C. E., Owen, W. M., Wang, T. C., Williams, B. G., Yeomans, D. K., and Scheeres, D. J., "Determination of Shape, Gravity, and Rotational State of Asteroid 433 Eros," Icarus, 2001.

20 Poloni, T., Kalabic, U., McDonough, K., and Kolmanovsky, I., "Disturbance canceling control based on simple input observers with constraint enforcement for aerospace applications," Proceedings of Conference on Control Applications, 2014, pp. 158-165.

${ }^{21}$ Kolmanovsky, I., Kalabic, U., and Gilbert, E., "Developments in constrained control using reference governors," Proceedings of the IFAC Conference on Nonlinear Model Predictive Control (NMPC), 2012, pp. 282-290.

${ }^{22}$ Gilbert, E. and Ong, C., "Constrained linear systems with hard constraints and disturbances: An extended command governor with large domain of attraction," Automatica, Vol. 47, No. 2, 2011, pp. 334-340.

${ }^{23}$ Gilbert, E. and Ong, C., "An Extended Command Governor for Constrained Linear Systems with Disturbances," Proceedings of Joint 48th IEEE Conference on Decision and Control and 28th Chinese Control Conference, 2009.

${ }^{24}$ Wächter, A. and Biegler, L. T., "On the implementation of an interior-point filter line-search algorithm for large-scale nonlinear programming," Mathematical programming, Vol. 106, No. 1, 2006, pp. 25-57.

${ }^{25}$ Duff, I. S., "MA57 - a code for the solution of sparse symmetric definite and indefinite systems," ACM Transactions on Mathematical Software (TOMS), Vol. 30, No. 2, 2004, pp. 118-144.

${ }^{26}$ Grant, M., Boyd, S., and Ye, Y., "CVX: Matlab software for disciplined convex programming," 2008. 
This article has been cited by:

1. Taylor Reynolds, Mehran Mesbahi. Small Body Precision Landing via Convex Model Predictive Control . [Citation] [PDF] [PDF Plus] 